\title{
Excess abdominal fat is associated with cutaneous allodynia in individuals with migraine: a prospective cohort study
}

Ane Mínguez-Olaondo 1,2, Iván Martínez-Valbuena 1,3,4 , Sonia Romero ${ }^{5,6}$, Gema Frühbeck ${ }^{4,5,6}$, María Rosario Luquin ${ }^{1,3,4}$, Eduardo Martínez-Vila ${ }^{1,4}$ and Pablo Irimia ${ }^{1,4^{*}}$ (i)

\begin{abstract}
Objective: To investigate the specific relationship between cutaneous allodynia (CA) and the percentages of body fat (BF) and abdominal fat in migraineurs. Additionally, we compared serum levels of inflammatory biomarkers in patients with and without CA.

Background: Excess abdominal fat might facilitate progressive changes in nociceptive thresholds causing central sensitization, clinically reflected as CA, which could drive migraine progression.

Methods: This prospective cohort study included 80 patients with migraine (mean age 39 years, $81.2 \%$ female) and 39 non-migraine controls. We analysed each participant's height, body weight, and body mass index (BMI). The amount and distribution of BF was also assessed by air displacement plethysmography (ADP) and ViScan, respectively. We analysed serum levels of markers of inflammation, during interictal periods.

Results: We studied 52 patients with episodic migraine (EM) and 28 with chronic migraine (CM). Of the 80 patients, $53(53.8 \%)$ had CA. Migraineurs with CA had a higher proportion of abdominal fat values than patients without CA $(p=0.04)$. The independent risk factors for CA were the use of migraine prophylaxis (OR 3.26, 95\% Cl [1.14 to 9.32]; $p=0.03)$, proportion of abdominal fat (OR 1.13,95\% Cl [1.01 to 1.27]; $p=0.04)$, and presence of sleep disorders (OR $1.13,95 \% \mathrm{Cl}[00.01$ to 1.27$] ; p=0.04)$. The concordance correlation coefficient between the ADP and BMI measurements was 0.51 ( 0.3681 to 0.6247 ). CA was not correlated with the mean plasma levels of inflammatory biomarkers.
\end{abstract}

Conclusions: There is a relation between excess abdominal fat and CA. Abdominal obesity might contribute to the development of central sensitization in migraineurs, leading to migraine chronification.

Keywords: Cutaneous allodynia, Body fat, Body composition, BMI, Abdominal fat, Inflammation, Biomarker, Migraine chronification, Risk factors

\section{Background}

Migraine can evolve from an episodic to a chronic form at a rate of approximately $3 \%$ per year [1], and obesity is one of the most relevant modifiable risk factors for chronification [2-4]. The mechanisms underlying the association between obesity and migraine progression are not yet completely clear, but several lines of evidence

\footnotetext{
* Correspondence: pirimia@unav.es

Department of Neurology, Clínica Universidad de Navarra, Pío XII 36, 31008 Pamplona, Spain

${ }^{4}$ Navarra's Health Research Institute (IDISNA), Pamplona, Spain

Full list of author information is available at the end of the article
}

suggest that obesity may facilitate progressive changes in nociceptive thresholds, causing central sensitization and leading to migraine chronification $[2,3,5]$.

Overweight and obesity, according to the World Health Organization (WHO), are defined as abnormal or excessive fat accumulation that presents a risk to health. It has been suggested that the quantity and distribution of adipose tissue are crucial to understanding the association between obesity, defined as excess body fat (BF), and migraine progression [6, 7]. Body mass index (BMI) is traditionally used to estimate $\mathrm{BF}$, although, this measurement technique is unable to distinguish between lean 
body fat and fat mass and does not provide information about the distribution of adipose tissue [8-11]. Nevertheless, it may be possible to accurately assess BF and its distribution by air displacement plethysmography (ADP) and ViScan, respectively [7, 12]. In addition, obesity (and especially excess abdominal fat) is associated with an increase in chronic systemic inflammation [13] which may contribute to central sensitization $[11,14,15]$. Therefore, studying body composition measurements and circulating inflammatory mediators in migraineurs may provide a better understanding of the relationship between obesity and migraine $[6,11]$.

Enhanced central sensitization is thought to indicate an increased risk of migraine chronification [4]. Cutaneous allodynia (CA) is considered a clinical marker of central sensitization and is regarded as the perception of pain in response to non-noxious skin stimulation [12]. The presence of CA can be assessed with a simple validated questionnaire, the Allodynia Symptom Checklist12 or ASC-12 [16]. Several factors have been associated with an increased risk of CA in migraine patients, including female sex, high BMI values, the number of migraine days, comorbid depression and medication overuse $[4,12,16,17]$. However, no information is available about the influence of $\mathrm{BF}$, specifically increased abdominal fat, on CA.

Thus, we hypothesized that excess abdominal fat could play a role in migraine chronification by inducing central sensitization, which is clinically reflected as CA. This study aimed to investigate the relationship of $\mathrm{BF}$ and abdominal fat with CA in migraine patients. Additionally, we compared serum levels of inflammatory biomarkers in patients with and without CA.

\section{Patients and methods \\ Study design}

This was a prospective cohort study. We recruited a cohort of individuals with migraine and matched them to non-migraine controls, and both were examined for differences in fat related to the presence or absence of allodynia and levels of selected inflammatory biomarkers.

\section{Participants}

We included 80 patients with migraine and 39 nonmigraine controls. Patients were recruited from regular visitors to our clinic. The participants were adults aged 18 to 65 years. Patients attending our Neurology Department during this period were invited to participate in the study. All patients filled out a migraine headache diary for 1 month to establish the migraine frequency. Patients were diagnosed with episodic migraine (EM) or chronic migraine $(\mathrm{CM})$ according to the International Classification of Headache Disorders (ICHD-III) criteria [18]. The exclusion criteria were as follows: (a) severe systemic disease or disease that could alter the measurements of inflammatory mediators, including: active infection, recent myocardial infarction (within 3 months), recent surgery (within 3 months), renal failure, severe liver disease, or inflammatory or neoplastic disease; (b) immunosuppressed patients requiring treatment with steroids or immunosuppressants, HIV-infected patients, people with addiction to drugs or alcohol; (c) patients who had recently started (in the prior 4 weeks) preventive migraine treatment or consumed opioids; (d) pregnancy or breastfeeding status; (e) patients with major psychiatric disorders or intellectual disability; and (f) morbidly obese patients according to BMI criteria. A complete medical record, including personal and family history, was available for all the participants, and their physical examination and neuroimaging results (when appropriate) were normal in every case. A total of 39 non-migraineur individuals matched for age, sex, and BMI, without a personal or family history of migraine headaches, and who also complied all the exclusion criteria were recruited from among the hospital personnel as a control group. None of them had chronic pain conditions. All the participants provided written informed consent to participate prior to their enrolment in the study. This work was approved by the local research ethics committee and was conducted in accordance with the Declaration of Helsinki.

After a screening visit, eligible individuals were invited to complete a structured questionnaire designed to obtain demographic and clinical data such as age, sex, type of migraine (episodic or chronic), duration of the disease (years), frequency of attacks per month, analgesic consumption in the last month (no opioids were used), use of migraine prophylaxis, and different body composition parameters (height, weight, BMI, abdominal fat by ViScan or body fat percentage). Patients completed a thorough history about the presence of depressive and anxiety symptoms, and sleep problems (insomnia, restless legs syndrome, sleep apnoea and excessive daytime sleepiness). Patients with suspicion of anxiety or depression during the clinical interview were referred to Psychiatry. The Structured Clinical Interview for Statistical Manual of Mental Disorders, Fifth Edition (DSM-5) was used to identify and characterize anxiety and depression. A diagnosis of depression, anxiety or sleep disorder was also made in patients who had received a previous medical diagnosis, used anti-depressants or anxiolytics (with indication for depression or anxiety), or used treatments for sleep disorders. In addition, the Migraine Disability Assessment (MIDAS) [19], the Headache Impact Testshort form $^{\text {Tx }}$ (HIT-6) [20], and the 12-item Allodynia Symptom Checklist (ASC-12) questionnaires [16] were also completed. The protocol included blood extraction in a subset of patients and all controls. All data were fully anonymised prior to analysis for this study. 


\section{Anthropometric and body composition measurements}

All anthropometric and body composition measurements were obtained on the same day. Height was measured to the nearest $0.1 \mathrm{~cm}$ with a Holtain stadiometer (Holtain Ltd., Crymych, UK) and body weight was measured to the nearest $0.1 \mathrm{~kg}$ with a calibrated ADP electronic scale. Each participant's BMI was calculated according to WHO recommendations [21, 22].

Visceral and abdominal adiposity were quantified with an abdominal BIA ViScan device (Tanita AB-140, Tanita Corp., Tokyo, Japan). This device measures total abdominal adiposity (percentage abdominal fat [range: 0 to $75 \%]$ ), including subcutaneous abdominal and intraabdominal adipose tissue expressed as 'visceral fat' (range: 1 to 59 arbitrary units [a.u.]). As stated by the manufacturer, visceral fat a.u. obtained by the ViScan multiplied by 10 correspond to intra-abdominal adipose tissue measured by computed tomography in $\mathrm{cm}^{2}$.

Body density was calculated by ADP (Bod-Pod ${ }^{\oplus}$, Life Measurements, Concord, California, USA), to estimate fat-free mass and fat mass. The percentage of BF (\%BF) was calculated from the body density using the Siri equation. Based on the most frequently used criteria in the literature [23], the \%BF cut-off points used to define individuals who were overweight were: $20.1 \%$ to $24.9 \%$ for men and $30.1 \%$ to $34.9 \%$ for women; for obese participants it was: $25.0 \%$ or more for men and $35.0 \%$ or more for women. True obesity was determined as the proportion of abdominal fat mass, which was distinguished from the peripheral and appendicular fat mass as a measure of abdominal adiposity.

\section{Blood determinations}

Venous blood serum samples were collected in chemisty test tubes in the morning after an overnight fast to avoid potential confounding influences resulting from hormonal rhythmicity, centrifuged at $3000 \mathrm{~g}$ for $15 \mathrm{~min}$, and immediately frozen and stored at $80^{\circ} \mathrm{C}$. Commercially available ELISA kits were used to assess circulating levels of pro-inflammatory cytokines including interleukin (IL-6; RayBiotech, Inc., Norcross, GA), and tumour necrosis factor-alpha (TNF- $\alpha$; R\&D Systems, Abingdon, UK) according to the manufacturer's instructions. The intra- and inter-assay coefficients of variation were: less than $10 \%$ and $12 \%$ for IL-6, and $5.4 \%$ and $8.3 \%$ for TNF$\alpha$ respectively. All samples and calibrator controls were run in duplicate and the average values were calculated blinded to the clinical data.

\section{Assessment of cutaneous allodynia}

The ASC-12, which includes 12 questions about the frequency of allodynia symptoms in association with headache attacks, was used to assess CA [16]. The responses were scored from 0 to 2, where: $0=$ 'never', 'rarely', or 'does not apply to me'; $1=$ 'less than half the time'; and $2=$ 'half the time or more'. The ASC-12 yields overall scores ranging from 0 to 24 and defines the following categories: no allodynia ( 0 to 2 points), mild allodynia ( 3 to 5 points), moderate allodynia (6 to 8 points), and severe allodynia ( 9 points or more). Previous validation analysis suggested that CA has three factors: thermal, mechanical static, and mechanical dynamic [16]. The thermal factor, reflects pain sensitivity to heat and cold and includes five items (shaving your face, taking a shower, resting your face or head on a pillow, exposure to heat, and exposure to cold). The mechanical static factor is composed of five items (wearing eyeglasses, wearing contact lenses, wearing earrings, wearing a necklace, and wearing tight clothing) and reflects fixedpoint pressure. The mechanical dynamic factor comprises two items (combing your hair and pulling your hair back) and reflects a more dynamic pressure across an area of skin [16].

\section{Statistics}

Before commencing the study, we determined that, assuming a migraineur/control proportion of $2: 1$, a standard deviation of 7.5 in both groups, a significance level of 0.05 , and a power of 0.90 , a sample of at least 73 patients in the migraine group and 37 patients in the control group would be required to detect a mean difference exceeding or equal to 5 abdominal fat units. To account for potential dropouts, we included $80 \mathrm{mi}$ graine patients and 39 non-migraine volunteers.

All statistical analyses were performed using SPSS software, version 15.0.1 (SPSS, Chicago, IL, USA), and $p$ values lower than 0.05 were considered statistically significant. The data were summarized using frequency counts and descriptive statistics, and presented as the mean $\pm S D$. The ASC scores were evaluated as indicated above. The Kolmogorov-Smirnov test was used to test the normality of the distributions. The means of normally distributed independent samples were compared using two-tailed Student's $t$-test. Nonnormally distributed data were compared with Mann-Whitney $U$ tests. Categorical variables were compared using the Chisquare test and Fisher's exact test, as required.

We compared the correlation and concordance of the $\%$ BF classification versus the BMI classification using Lin's concordance coefficients ( $\rho c)$. We used Lin's coefficient of correlation instead of Pearson's, because the latter estimates the general linear association and not the association specific to the equivalence line [24]. The $\rho c$ reproducibility was considered almost perfect (substantial) when values exceeded 0.99; values between 0.95 and 0.99 they were moderate; and those below 0.90 were poor [11].

A logistic regression model for the univariate analysis and multivariate multiple regression model for the 
multivariate analyses were used to test the association between $\mathrm{CA}$, as the dependent variable, and different study variables. Variables were entered into the model by the backward strategy. The multivariate analysis included factors associated with success in the univariate analysis at $p \leq 0.1$. Moreover, a supplementary analysis was performed with the pro-inflammatory cytokines and another substituting abdominal fat values for obesity and body fat values. The model was adjusted by age, MIDAS results, HIT- 6 results and years with migraine. To ensure that collinearity was not a problem in the regression analysis, we the used variance inflation factor.

\section{Results}

A total of 119 Caucasian individuals were included in our analyses: 80 migraine patients (52 with EM and 28 with $\mathrm{CM}$ ) and 39 nonmigraine controls. The mean age of the migraineurs was $39 \pm 10$ years, and $81.2 \%$ were female ( $37 \pm 8$ years for EM and $41 \pm 12$ years for CM, $78.8 \%$ and $85.7 \%$ female, respectively). The mean age of the nonmigraine controls was $41 \pm 10$ years, and $79.5 \%$ were female. There was no significant difference in age, sex, BMI, or body composition between the patients and controls (Table 1).

In this cohort, 43 migraine patients (53.8\%) had CA during their migraine attacks. Patients with CA had higher subcutaneous abdominal fat $(35.7 \pm 8.7)$, compared to patients without CA $(32.9 \pm 7.5, p=0.04)$ and controls $(29.8 \pm 12.2, p=0.04)$ (Fig. 1). However, there were no significant differences in subcutaneous abdominal fat between the whole sample of migraine patients and controls $(p=0.09)$. The appearance of CA in migraineurs was also associated with older age $(p=0.05)$, higher HIT-6 (62 \pm 7 vs. $58 \pm 9 ; p=0.03)$ and MIDAS scores ( $54 \pm 57$ vs. $29 \pm 40 ; p=0.05)$, an increase in analgesic consumption ( $11 \pm 8$ vs. $7 \pm 7 ; p=0.03)$, the use of migraine prophylaxis $(59.5 \%$ vs. $32.4 \% ; p=0.02)$, and sleep disorders ( $69.8 \%$ vs. $43.2 \%, p=0.02$; Table 2$)$. BodPod analysis indicated a trend towards higher \%BF scores in patients with allodynia. None of the variables analysed significantly differed in relation to the type or intensity of CA. Thus, independent risk factors for the development of CA were the use of migraine prophylaxis (OR 3.26, 95\% CI [1.14 to 9.32]: $p=0.03$ ), abdominal fat (OR 1.13, 95\% CI [1.01 to 1.27]: $p=0.04$ ), and sleep disorders (OR 1.13, 95\% CI [0.01 to 1.27]: $p=0.04$ ).

The concordance coefficient between \%BF and BMI measurements was 0.51 (0.3681 to 0.6247$)$ with a Pearson correlation coefficient of $0.72(0.57$ to $0.82 ; p<0.0001)$, while in the controls the concordance coefficient between the ADP and BMI measurements was 0.04 ( -0.1396 to 0.2204 ). With a sample of 52 patients in the episodic group and 28 in the CM group, this analysis had $82 \%$ statistical power to detect a difference of 5 units in abdominal fat between the two groups at a significance level of 0.05 . In addition, the Spearman correlation coefficient was 0.61 , indicating a moderate level of correlation between the waist-to-hip ratio and abdominal fat.

We performed blood tests on 56 of the 80 patients included, of these 29 were obese, 12 were overweight, 14 were normal weight and 1 was underweight. The mean TNF- $\alpha$ levels were significantly higher in patients who had migraine (1.19 vs. $0.92 \mathrm{pg} / \mathrm{mL} ; p<0.001$ ), and IL-6 levels were almost significantly different $(9.23$ vs. 5.47 $\mathrm{pg} / \mathrm{mL} ; p=0.05)$ compared to these parameters in the controls. We did not find statistically significant differences between the levels of TNF and IL- 6 in patients with CA compared to non-CA patients or controls.

\section{Discussion}

In the present study, increased subcutaneous abdominal fat was independently associated with CA. This novel finding suggests that excess abdominal fat might contribute to the development of central sensitization in migraineurs. Published research about the relationship between CA and total body adiposity or abdominal adiposity is currently limited. An association between high BMI and allodynia has been suggested [12], although this relationship was not confirmed in a subsequent study [6]. This discrepancy might reflect the dependence

Table 1 Anthropometric characteristics of migraine patients versus non-migraine controls

\begin{tabular}{llll}
\hline Variables & Non-migraine controls $(n=39)$ & Migraineurs $(n=80)$ & $p$-value \\
\hline Age (years) & $41 \pm 10$ & $39 \pm 10$ & 0.56 \\
Female (\%) & $79.5 \%$ & $81.2 \%$ & $0.82^{\mathrm{b}}$ \\
Height $(\mathrm{m})$ & $1.70 \pm 0.10$ & $1.65 \pm 0.09$ & 0.84 \\
Weight $(\mathrm{kg})$ & $70.0 \pm 16.0$ & $64.3 \pm 11.7$ & 0.07 \\
BMl $\left(\mathrm{kg} / \mathrm{m}^{2}\right)$ & $25.5 \pm 4.6$ & $23.7 \pm 3.5$ & 0.05 \\
Body composition - ViScan abdominal fat & $29.8 \pm 12.2$ & $34.4 \pm 8.3$ & 0.09 \\
Body fat percentage & $32.4 \pm 10.5$ & $31.9 \pm 8.7$ & $0.54^{\mathrm{b}}$ \\
\hline
\end{tabular}

$B M I$ body mass index

${ }^{a}$ Two-tailed independent samples, Student's t/Mann-Whitney $U$ tests

${ }^{\mathrm{b}}$ Chi-squared test 


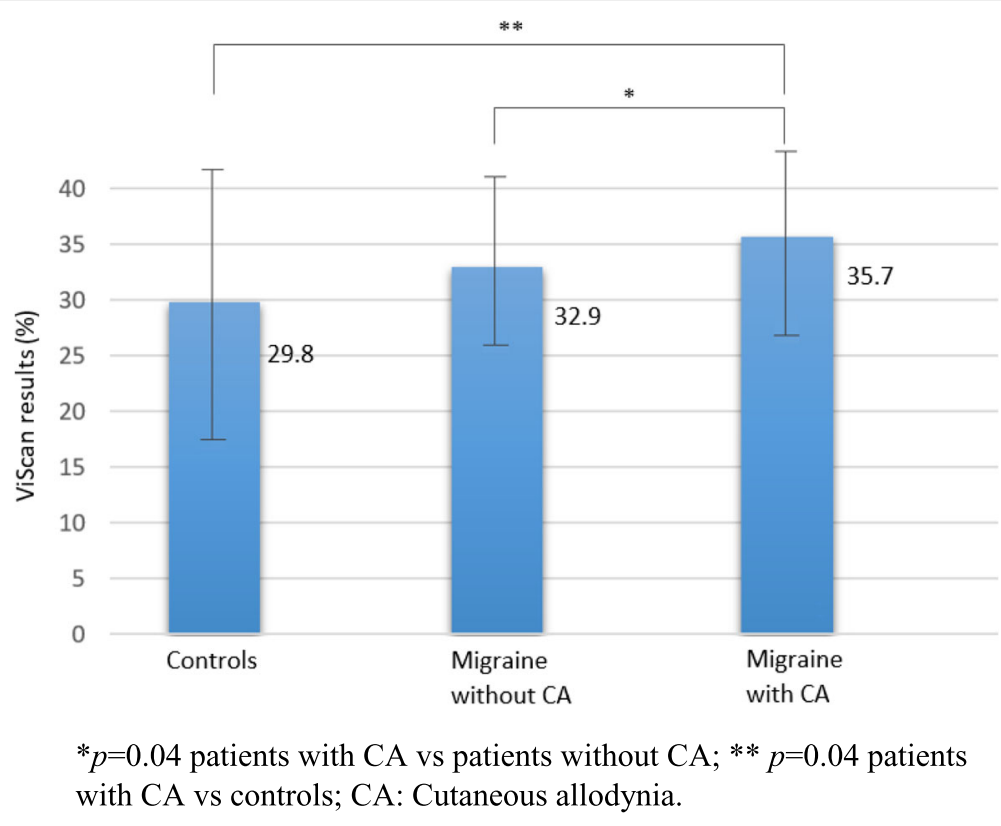

Fig. 1 Abdominal fat in patients with or without cutaneous allodynia. ${ }^{*} p=0.04$ patients with CA vs patients without $C A$; ${ }^{* *} p=0.04$ patients with CA vs controls; CA: Cutaneous allodynia

Table 2 Anthropometric and clinical characteristics in migraine patients with and without cutaneous allodynia

\begin{tabular}{|c|c|c|c|}
\hline Variables & Migraineurs with CA $(n=43)$ & Migraineurs without CA $(n=37)$ & $p$-value ${ }^{a}$ \\
\hline Age (years) & $41 \pm 11$ & $37 \pm 9$ & 0.05 \\
\hline Female (\%) & $86.5 \%$ & $75.7 \%$ & 0.24 \\
\hline Height (m) & $1.64 \pm 0.09$ & $1.65 \pm 0.08$ & 0.52 \\
\hline Weight (kg) & $64.7 \pm 11.2$ & $64.1 \pm 12.4$ & 0.79 \\
\hline BMI $\left(\mathrm{kg} / \mathrm{m}^{2}\right)$ & $24.1 \pm 3.9$ & $23.4 \pm 3.1$ & 0.42 \\
\hline Body composition - ViScan abdominal fat & $35.7 \pm 8.7$ & $32.9 \pm 7.5$ & 0.04 \\
\hline Body fat percentage & $32.6 \pm 9.4$ & $31.1 \pm 7.78$ & 0.07 \\
\hline HIT-6 & $62.68 \pm 7.19$ & $58.47 \pm 9.03$ & 0.03 \\
\hline MIDAS & $54.83 \pm 57.83$ & $29.03 \pm 40.09$ & 0.05 \\
\hline Migraine evolution (years) & $9.9 \pm 8.6$ & $9.7 \pm 10.3$ & 0.03 \\
\hline Frequency (attacks per month) & $9.49 \pm 8.70$ & $5.25 \pm 5.17$ & 0.23 \\
\hline 1-9 days per month & $23(53.5 \%)$ & $28(75.0 \%)$ & \\
\hline 10-14 days per month & $1(2.3 \%)$ & $1(2.8 \%)$ & $0.31^{b}$ \\
\hline$>15$ days per month & $19(44.2 \%)$ & $8(22.2 \%)$ & \\
\hline Analgesic consumption (last month) & $11.32 \pm 8.01$ & $7.36 \pm 7.39$ & 0.03 \\
\hline Triptan consumption (last month) & $4.47 \pm 6.69$ & $3.05 \pm 6.51$ & 0.464 \\
\hline Medication overuse & $21(48.8 \%)$ & $10(27.0 \%)$ & $0.726^{b}$ \\
\hline Use of migraine prophylaxis & $25(59.5 \%)$ & $12(32.4 \%)$ & $0.02^{b}$ \\
\hline Depression (\%) & $16.2 \%$ & $10.8 \%$ & $0.49^{b}$ \\
\hline Anxiety disorders (\%) & $37.2 \%$ & $37.5 \%$ & $0.95^{b}$ \\
\hline Sleep disorders (\%) & $69.8 \%$ & $43.2 \%$ & $0.02^{b}$ \\
\hline
\end{tabular}

Anxiety and mood disorders as well as sleep disorders were self-reported

$B M I$ body mass index, CA cutaneous allodynia, HIT-6 6-item Headache Impact Test, MIDAS Migraine Disability Assessment

${ }^{\text {a }}$ Two-tailed independent samples Student's $t /$ Mann-Whitney U tests

${ }^{\mathrm{b}}$ Chi-squared test 
of $\mathrm{CA}$ on excess abdominal BF because the use of BMI for diagnosing obesity may be inaccurate. In this work, several migraine patients with a normal BMI actually had excess BF or abdominal fat and BMI underestimated obesity compared to ADP. Thus, our findings reinforce the notion that focusing on body composition rather than BMI is preferable when studying the association between migraine and obesity $[6,11]$ because, according to our results and other observations [11,25], the use of BMI for the diagnosis of obesity might be inaccurate. The relevance of abdominal fat in the risk of migraine progression has been suggested previously [6, 26-28]. In accordance with our findings, the prevalence of migraine was higher in individuals aged $20-55$ years with abdominal obesity and this association was independent of overall body obesity in women (28). Furthermore, CM has already been associated with metabolic syndrome, a cluster of common abnormalities that include abdominal obesity [29].

The mechanisms that underlie the association between increased abdominal fat and CA in migraine patients are not yet well understood. One possible explanation is that excess abdominal $\mathrm{BF}$, which is metabolically more active, may promote chronic low-grade systemic inflammation [11]. Thus, the release of different inflammatory mediators might decrease the threshold for the onset of a migraine attack and contribute to central sensitization [14, 30-33]. We found that the blood serum levels of only TNF- $\alpha$, but not IL-6, were significantly higher in migraine patients than in the controls. These findings may suggest that the increase in BF can intensify the neurovascular inflammatory response in migraine, promoting the sensitization of central neurons with an increased risk of progression, as suggested by Di Renzo L, et al. [11]. Thus, in addition to inflammation, dysfunction in the orexin pathways and the release of neuropeptides associated with pain transmission, (such as calcitonin gen-related peptide-CGRP-) might be involved in the development of central sensitization and migraine progression [34].

We also found that CA was associated both with the use of preventive medications and disrupted sleep, but not with medication overuse. The use of migraine prophylaxis probably reflects the fact that these patients had more frequent migraine attacks and explains this association. Although drug-induced weight gain is a side effect associated with some of the medications prescribed for migraine prophylaxis, in this cohort, patients were treated with different preventive drugs, including topiramate which is associated with weight loss. Taken together, our findings, in concordance with previous observations [27], do not suggest that the use of migraine prophylaxis influences the distribution of adipose tissue. Indeed, our data agree with previous publications in which headache frequency $[4,12,35]$ and poorer sleep quality [36] were both associated with $\mathrm{CA}$ in patients who had migraines. Overuse of antimigraine medications may induce central sensitization [17]. In our sample, the presence of medication overuse was more frequent in patients with CA, but the difference did not achieve statistical significance (Table 2).

$\mathrm{CA}$ is also more frequent in headache patients with major depression and in different populations of migraineurs, depression was independently associated with CA [12, 37-39]. In our cohort, depression was more frequent in patients with $\mathrm{CA}$, yet the difference did not achieve statistical significance.

Our findings may have clinical implications. Excess abdominal fat, even in patients with a normal BMI, is associated with a higher risk of central sensitization, supporting the recommendation of fat loss in such individuals. Epidemiological research shows a link between obesity and the risk of migraine [40] or migraine progression $[2,3$, 41]. Different studies have demonstrated a decrease in migraine frequency after weight loss, either by dietary modifications [42, 43] or bariatric surgery [44, 45]. As such, weight loss is generally recommended for obese and overweight migraine patients [27, 46]. If excess abdominal fat is the main driver of migraine progression, further studies are needed to test the impact of abdominal fat reduction on migraine frequency and CA reduction.

One of the main strengths of this study is the accurate diagnosis of obesity based on detailed information of body composition to distinguish between fat free mass and fat mass and the distribution of adipose tissue. Furthermore, the individuals in this cohort received a correct migraine diagnosis according to the most recent IHS criteria, and all patients were treated by a consultant neurologist attached to a headache unit. The possible limitations of this study include the fact that the subjects were recruited from a headache clinic, excluding elderly patients, and thus the cohort may not be fully representative of migraine patients in other clinical settings. Additionally, psychiatric comorbidities and insomnia are more prevalent in patients with $\mathrm{CM}$ and both conditions are associated with CA. Although we directly asked our patients about their mood, anxiety symptoms and sleep problems, during the clinical interview, we did not use any formal questionnaire to diagnose depression, anxiety or the quality of sleep. In addition, we study the inflammatory biomarkers in a subgroup of patients. It is possible that larger studies may have identified differences in TNF- $\alpha$ and IL- 6 levels between patients with and without CA. Finally, another possible limitation is that many of our patients who had migraines were receiving daily prophylactic treatments which could have influenced the levels of pro-inflammatory mediators and the appearance of CA. However, for ethical reasons, migraine prophylaxis should not be discontinued. 


\section{Conclusions}

In our cohort, we found that excess abdominal fat is associated with CA, a marker of central sensitization in patients with migraine. The distribution of adipose tissue appeared to strongly influence the association between obesity and the risk of migraine chronification. Further research into the relationship between BF distribution and the risk of migraine progression is warranted.

\section{Abbreviations}

ADP: Air displacement plethysmography; ASC: Allodynia Symptom Checklist; BF: Body fat; BMI: Body Mass Index; CA: Cutaneous allodynia;

CGRP: Calcitonin gen-related peptide; Cl: Confidence intervals; CM: Chronic migraine; EDQL: Today's quality of life; EM: Episodic migraine; HIT-6: the sixitem Headache Impact Text; HIV: Human immunodeficiency viruses; ICHDIII: International Classification of Headache Disorders; IL-6: Interleukin 6; MIDAS: Migraine Disability Assessment Questionnaire; OR: Odds ratio; Rc: Lin's concordance correlation coefficients; SD: Standard deviation; TNFa: Alfa-Tumor necrosis factor; WHO: World Health Organization

\section{Acknowledgments}

Allergan Inc. funded editorial assistance and statistical advice.

\section{Authors' contributions}

AMO made substantial contributions to the acquisition, analysis and drafted the manuscript. IMV made substantial contributions to the conception, design and interpretation of data of the work. SR made substantial contributions to the acquisition of data. GF have made substantial contributions to the conception, design of the work and revised the manuscript. MRL made substantial contributions to the design of the work, the acquisition, and interpretation of data and revised the manuscript. EMV made substantial contributions to the design of the work, the acquisition, and interpretation of data. PI have made substantial contributions to the conception, design of the work; the acquisition, analysis, and interpretation of data; and have drafted the work and substantively revised it. All authors read and approved the final manuscript.

\section{Funding}

Neither honoraria nor payments were made for authorship. All authors met the ICMJE authorship criteria.

\section{Availability of data and materials}

The datasets used and/or analysed during the current study are available from the corresponding author on reasonable request.

\section{Ethics approval and consent to participate}

See "Participants" section above.

\section{Consent for publication}

See "Participants" section above.

\section{Competing interests}

Ane Mínguez-Olaondo, Iván Martínez-Valbuena, Eduardo Martínez-Vila, Sonia Romero and Gema Frühbeck declare that they have no competing interests. María Rosario Luquin have received honoraria from TEVA, Zambon, AbbVie and Bial for activities unrelated to the work submitted. Pablo Irimia has received honoraria from Allergan, Novartis, Lilly and Teva Pharmaceuticals as a consultant and speaker.

\section{Author details}

'Department of Neurology, Clínica Universidad de Navarra, Pío XII 36, 31008 Pamplona, Spain. ²Department of Neurology, Hospital Universitario Donostia, San Sebastián, Spain. ${ }^{3}$ Regenerative Therapy Laboratory, Neurosciences Division, Center for Applied Medical Research (CIMA), University of Navarra, Pamplona, Spain. ${ }^{4}$ Navarra's Health Research Institute (IDISNA), Pamplona, Spain. ${ }^{5}$ Endocrinology and Nutrition Department, Clínica Universidad de Navarra, Pamplona, Spain. ${ }^{6}$ CIBER Fisiopatología de la Obesidad y Nutrición (CIBERobn), Instituto de Salud Carlos III, Madrid, Spain.
Received: 29 October 2019 Accepted: 29 January 2020

Published online: 04 February 2020

\section{References}

1. Lipton RB, Fanning KM, Serrano D, Reed ML, Cady R, Buse DC (2015) Ineffective acute treatment of episodic migraine is associated with newonset chronic migraine. Neurology. 84:688-695

2. Bigal ME, Lipton RB (2006) Modifiable risk factors for migraine progression Headache 46:1334-1343

3. May A, Schulte LH (2016) Chronic migraine: risk factors, mechanisms and treatment. Nat Rev Neurol 12:455-464

4. Louter MA, Bosker JE, van Oosterhout WP, van Zwet EW, Zitman FG, Ferrari MD et al (2013) Cutaneous allodynia as a predictor of migraine chronification. Brain. 136:3489-3496

5. Rossi HL, Luu AK, DeVilbiss JL, Recober A (2013) Obesity increases nociceptive activation of the trigeminal system. Eur J Pain 17:649-653

6. Recober A, Peterlin BL (2014) Migraine and obesity: moving beyond BMI. Future Neurol 9:37-40

7. Gómez-Ambrosi J, Catalán V, Rodríguez A, Andrada P, Ramírez B, Ibáñez P et al (2014) Increased cardiometabolic risk factors and inflammation in adipose tissue in obese subjects classified as metabolically healthy. Diabetes Care 37:2813-2821

8. Gomez-Ambrosi J, Salvador J, Silva C, Pastor C, Rotellar F, Gil MJ et al (2006) Increased cardiovascular risk markers in obesity are associated with body adiposity: role of leptin. Thromb Haemost 95:991-996

9. Lee SY, Gallagher D (2008) Assessment methods in human body composition. Curr Opin Clin Nutr Metab Care 11:566-572

10. Gomez-Ambrosi J, Silva C, Galofre JC, Escalada J, Santos S, Millan D et al (2012) Body mass index classification misses subjects with increased cardiometabolic risk factors related to elevated adiposity. Int J Obes (Lond) 36:286-294

11. Di Renzo L, Cammarano A, De Lorenzo A (2018) The missclassification of obesity affects the course of migraine. J Headache Pain 19:63

12. Bigal ME, Ashina S, Burstein R, Reed ML, Buse D, Serrano D et al (2008) Prevalence and characteristics of allodynia in headache sufferers: a population study. Neurology. 70:1525-1533

13. Panagiotakos DB, Pitsavos C, Yannakoulia M, Chrysohoou C, Stefanadis C (2005) The implication of obesity and central fat on markers of chronic inflammation: the ATTICA study. Atherosclerosis. 183:308-315

14. Kawasaki Y, Zhang L, Cheng JK, Ji RR (2008) Cytokine mechanisms of central sensitization: distinct and overlapping role of interleukin-1 beta, interleukin-6, and tumor necrosis factor-alpha in regulating synaptic and neuronal activity in the superficial spinal cord. J Neurosci 28:5189-5194

15. Wada N, Hirako S, Takenoya F, Kageyama H, Okabe M, Shioda S (2014) Leptin and its receptors. J Chem Neuroanat 61-62:191-199

16. Lipton RB, Bigal ME, Ashina S, Burstein R, Silberstein S, Reed ML et al (2008) Cutaneous allodynia in the migraine population. Ann Neurol 63:148-158

17. Coppola G, Curra A, Di Lorenzo C, Parisi V, Gorini M, Sava SL et al (2010) Abnormal cortical responses to somatosensory stimulation in medicationoveruse headache. BMC Neurol 10:126

18. Headache Classification Committee of the International Headache Society (IHS) The International Classification of Headache Disorders, 3rd edition. Cephalalgia. 2018:38:1-211

19. Stewart WF, Lipton RB, Kolodner KB, Sawyer J, Lee C, Liberman JN (2000) Validity of the migraine disability assessment (MIDAS) score in comparison to a diary-based measure in a population sample of migraine sufferers. Pain. 88:41-52

20. Kosinski M, Bayliss MS, Bjorner JB, Ware JE Jr, Garber WH, Batenhorst A et al (2003) A six-item short-form survey for measuring headache impact: the HIT-6. Qual Life Res 12:963-974

21. Physical status: the use and interpretation of anthropometry. Report of a WHO Expert Committee. World Health Organ Tech Rep Ser. 1995:854:1-452

22. Flegal KM, Carroll MD, Kuczmarski RJ, Johnson CL (1998) Overweight and obesity in the United States: prevalence and trends, 1960-1994. Int J Obes Relat Metab Disord 22:39-47

23. Okorodudu DO, Jumean MF, Montori VM, Romero-Corral A, Somers VK, Erwin PJ et al (2010) Diagnostic performance of body mass index to identify obesity as defined by body adiposity: a systematic review and metaanalysis. Int J Obes (Lond) 34:791-799

24. Lin $\amalg$ (2000) A note on the concordance correlation coefficient. Biometrics. 56:324-325 
25. De Lorenzo A, Soldati L, Sarlo F, Calvani M, Di Lorenzo N, Di Renzo L (2016) New obesity classification criteria as a tool for bariatric surgery indication. World J Gastroenterol 22:681-703

26. Verrotti A, Carotenuto M, Altieri L, Parisi P, Tozzi E, Belcastro V et al (2015) Migraine and obesity: metabolic parameters and response to a weight loss programme. Pediatr Obes 10:220-225

27. Santos IS, Goulart AC, Passos VM, Molina Mdel C, Lotufo PA, Bensenor IM (2015) Obesity, abdominal obesity and migraine: a cross-sectional analysis of ELSA-Brasil baseline data. Cephalalgia. 35:426-436

28. Peterlin BL, Rosso AL, Rapoport AM, Scher Al (2010) Obesity and migraine: the effect of age, gender and adipose tissue distribution. Headache. 50:5262

29. Rainero I, Govone F, Gai A, Vacca A, Rubino E (2018) Is migraine primarily a Metaboloendocrine disorder? Curr Pain Headache Rep 22:36

30. Gomez-Ambrosi J, Silva C, Galofre JC, Escalada J, Santos S, Gil MJ et al (2011) Body adiposity and type 2 diabetes: increased risk with a high body fat percentage even having a normal BMl. Obesity (Silver Spring) 19:1439-1444

31. Ji RR, Nackley A, Huh Y, Terrando N, Maixner W (2018) Neuroinflammation and central sensitization in chronic and widespread pain. Anesthesiology. 129:343-366

32. Gomez-Ambrosi J, Salvador J, Paramo JA, Orbe J, de Irala J, Diez-Caballero A et al (2002) Involvement of leptin in the association between percentage of body fat and cardiovascular risk factors. Clin Biochem 35:315-320

33. Rodriquez A, Ezquerro S, Mendez-Gimenez L, Becerril S, Fruhbeck G (2015) Revisiting the adipocyte: a model for integration of cytokine signaling in the regulation of energy metabolism. Am J Physiol Endocrinol Metab 309: E691-E714

34. Strother LC, Srikiatkhachorn A, Supronsinchai W (2018) Targeted Orexin and hypothalamic neuropeptides for migraine. Neurotherapeutics. 15:377-390

35. Dodick DW, Reed ML, Fanning KM, Munjal S, Alam A, Buse DC et al (2019) Predictors of allodynia in persons with migraine: results from the migraine in America symptoms and treatment (MAST) study. Cephalalgia. 39:873-882

36. de Tommaso M, Delussi M, Vecchio E, Sciruicchio V, Invitto S, Livrea P (2014) Sleep features and central sensitization symptoms in primary headache patients. J Headache Pain. 15:64

37. Fuller-Thomson E, Schrumm M, Brennenstuhl S (2013) Migraine and despair: factors associated with depression and suicidal ideation among Canadian Migraineurs in a population-based study. Depress Res Treat 2013:401487

38. Louter MA, Wardenaar KJ, Veen G, van Oosterhout WP, Zitman FG, Ferrari MD et al (2014) Allodynia is associated with a higher prevalence of depression in migraine patients. Cephalalgia. 34:1187-1192

39. Park SP, Seo JG, Lee WK (2015) Osmophobia and allodynia are critical factors for suicidality in patients with migraine. J Headache Pain. 16:529

40. Gelaye B, Sacco S, Brown WJ, Nitchie HL, Ornello R, Peterlin BL (2017) Body composition status and the risk of migraine: a meta-analysis. Neurology. 88 : 1795-1804

41. Bigal ME, Lipton RB (2006) Obesity is a risk factor for transformed migraine but not chronic tension-type headache. Neurology. 67:252-257

42. Lemstra M, Stewart B, Olszynski WP (2002) Effectiveness of multidisciplinary intervention in the treatment of migraine: a randomized clinical trial. Headache. 42:845-854

43. Verrotti A, Agostinelli S, D'Egidio C, Di Fonzo A, Carotenuto M, Parisi P et al (2013) Impact of a weight loss program on migraine in obese adolescents. Eur J Neurol 20:394-397

44. Bond DS, Vithiananthan S, Nash JM, Thomas JG, Wing RR (2011) Improvement of migraine headaches in severely obese patients after bariatric surgery. Neurology. 76:1135-1138

45. Novack V, Fuchs L, Lantsberg L, Kama S, Lahoud U, Horev A et al (2011) Changes in headache frequency in premenopausal obese women with migraine after bariatric surgery: a case series. Cephalalgia. 31:1336-1342

46. Chai NC, Bond DS, Moghekar A, Scher Al, Peterlin BL (2014) Obesity and headache: part II--potential mechanism and treatment considerations. Headache. 54:459-471

\section{Publisher's Note}

Springer Nature remains neutral with regard to jurisdictional claims in published maps and institutional affiliations.

Ready to submit your research? Choose BMC and benefit from:

- fast, convenient online submission

- thorough peer review by experienced researchers in your field

- rapid publication on acceptance

- support for research data, including large and complex data types

- gold Open Access which fosters wider collaboration and increased citations

- maximum visibility for your research: over $100 \mathrm{M}$ website views per year

At $\mathrm{BMC}$, research is always in progress.

Learn more biomedcentral.com/submissions 